\section{IRAK-M regulation and function in host defense and immune homeostasis}

\author{
Leah L.N. Hubbard, ${ }^{1}$ Bethany B. Moore ${ }^{2}$ \\ 'Graduate Program in Immunology; \\ ${ }^{2}$ Departments of Internal Medicine, \\ Microbiology and Immunology, University \\ of Michigan, Ann Arbor, USA
}

\section{Abstract}

Antigen presenting cells (APCs) of the innate immune system sense a wide range of pathogens via pattern recognition receptors (PRRs). Engagement of certain PRRs can induce production of pro-inflammatory mediators that facilitate effective clearance of pathogen. Toll-like receptors (TLRs) are a well described group of PRRs that belong to the TLR/Interleukin-1 receptor (IL-1R) superfamily. However, TLR/L-1R induction of pro-inflammatory mediators must be regulated to prevent excessive inflammation and tissue damage. One molecule of recent interest that is known to inhibit TLR/IL-1R signaling is interleukin-1 receptor associated kinase (IRAK)-M, also known as IRAK-3. IRAK-M is expressed in a number of immune and epithelial cells types, and through its inhibition of pro-inflammatory cytokine production, IRAK-M can regulate immune homeostasis and tolerance in a number of infectious and non-infectious diseases. Furthermore, use of IRAK-M deficient animals has increased our understanding of the importance of IRAK-M in regulating immune responsiveness to a variety of pathogens. Although IRAK-M expression is typically induced through TLR signaling, IRAK-M can also be expressed in response to various endogenous and exogenous soluble factors as well as cell surface and intracellular signaling molecules.

This review will focus on clinical scenarios in which expression of IRAK-M is beneficial (as in early sepsis) and those situations where IRAK-M expression is harmful to the host (as in cancer and following bone marrow transplant). There is strong rationale for therapeutic targeting of IRAK-M for clinical benefit. However, effective targeting will require a greater understanding of the transcriptional regulation of this gene.

\section{Introduction}

In response to invading pathogens, the host elicits an inflammatory response mediated initially by antigen presenting cells (APCs) of the innate immune system, such as macrophages and dendritic cells. Pathogen associated molecular patterns (PAMPs) on microbes, as well as certain endogenous proteins released from tissue damage, engage pattern recognition receptors (PRRs) expressed on the surface of APCs. Binding of these "danger signals" to PRRs can activate a number of signaling pathways to induce transcription and subsequent release of pro-inflammatory cytokines and chemokines. Consequently, this proinflammatory milieu recruits more innate immune cells to the site of infection and facilitates activation of the adaptive immune system for effective clearance of pathogen and resolution of tissue damage.

Toll-like receptors (TLRs) are a well described group of PRRs belonging to the TLR/Interleukin-1 receptor (IL-1R) or TIR superfamily. ${ }^{1}$ Both TLRs and IL-1Rs have a common intracellular TIR domain; however, TLRs have a leucine-rich repeat motif in the extracellular domain, while IL-1Rs have an immunoglobulin containing extracellular domain. Activation of TLRs and IL-1Rs recruits the adaptor molecule myeloid differentiation primary response gene 88 (MyD88) to their TIR domains. Members of the interleukin-1 receptor associated kinase (IRAK) family and various other proteins bind to MyD88 and form an adaptor complex that activates signaling pathways for induction of pro-inflammatory gene transcription (Figure 1). However, induction of pro-inflammatory mediators via TLR/IL$1 \mathrm{R}$ signaling must be tightly regulated to prevent damage to tissues and diseases associated with excessive inflammation, such as cancer, sepsis, and autoimmunity.

A large number of molecules have been identified, that negatively regulate TLR/IL-1R signaling. A list of these molecules and their known inhibitory functions are summarized in Table 1 and are reviewed extensively by Wang et $a l .{ }^{1}$ Of particular interest is the role of the inhibitory IRAK family member, IRAK-M，also known as IRAK-3. The current review will focus on the regulation of IRAK-M and its role in host defense, endotoxin tolerance, and immune homeostasis. In terms of therapeutic applications, targeting IRAK-M may offer some benefit to improve innate immune function in immunosuppressed patients. Appropriate targeting will be critical, however, to prevent damage due to unchecked inflammation.

\section{IRAK-M structure, function and expression pattern}

\section{Structure and function}

Human IRAK-M was first characterized in 1999 by Wesche et al. ${ }^{2}$ The IRAK-M gene is
Correspondence: Bethany B. Moore, Department of Internal Medicine, Microbiology and Immunology, University of Michigan, 4053 BSRB, 109 Zina Pitcher Pl., Ann Arbor, MI 48109-2200, USA. Tel. + 1.734.6478378 - Fax: +1.734.615-2331.

E-mail: bmoore@umich.edu

Key words: sepsis, immune regulation, inflammation.

Contributions: LLNH reviewed the literature and wrote the review article; BBM revised and edited the manuscript.

Conflict of interest: the authors report no conflicts of interest.

Acknowledgements: this work was supported by NIH grant AI065543.

Received for publication: 12 May 2010

Revision received: 29 June 2010.

Accepted for publication: 29 June 2010.

This work is licensed under a Creative Commons Attribution 3.0 License (by-nc 3.0).

CC Copyright L.L.N. Hubbard and B.B. Moore, 2010 Licensee PAGEPress, Italy

Infectious Disease Reports 2010; 2:e9

doi:10.4081/idr.2010.eg

located on human chromosome 12 at position 12q14.1-12q15 and encodes a $68 \mathrm{kDa}$ protein. All IRAKs (IRAK-1, -2, -M, and -4) have a conserved N-terminal death domain for MyD88 TIR-TIR interaction and a central serine/threonine kinase domain consisting of 12 subdomains. However, only IRAK- 1 and -4 have a functional catalytic site containing a critical aspartate residue in kinase subdomain VI. This residue is an asparagine and serine in IRAK-2 and -M respectively, rendering these kinases inactive. ${ }^{2}$ IRAK-2 has four splice variants, and two of these isoforms are inhibitory of TLR signaling. ${ }^{4}$ The murine IRAK-M homologue shows $71 \%$ sequence identity to human IRAK-M and has similar limitations in kinase activity. ${ }^{5}$ It is now known that IRAK-M negatively regulates TLR/L-1R signaling by binding the MyD88 adaptor complex. ${ }^{6}$

\section{Expression pattern in tissues}

Human IRAK-M expression is generally confined to monocytes and macrophages (Table 2 ), and its expression is induced during macrophage maturation and TLR/L-1R signaling. ${ }^{2,7}$ Tissue-specific mRNA expression in human brain, liver, thymus and small intestine has not been detected. ${ }^{2}$ However, recent studies by Balaci et al. show IRAK-M protein expression in lung epithelial cells of asthmatic patients. ${ }^{8}$ This is consistent with murine studies examining IRAK-M expression in the lung, 
where alveolar epithelial cells express IRAK-M under both basal and inflammatory conditions. $^{9,10}$

Murine IRAK-M has a broader expression pattern compared to human IRAK-M; it has been detected not only in monocytes and macrophages but also in neutrophils, ${ }^{11}$ the NIH 3T3 fibroblast cell line, ${ }^{5} \mathrm{~B}$ cells, ${ }^{12}$ and epithelial cells of the intestine, lung, and intrahepatic bilary ducts. ${ }^{9,10,13,14}$ Furthermore, IRAK-M mRNA has been detected in various tissues, with highest expression in the thymus and liver and relatively lower expression levels in the heart, brain, spleen, and kidney. ${ }^{5}$ It is not clear whether this is related to the presence of tissue-specific macrophages or epithelial cells that are known to express IRAK-M.

\section{IRAK-M regulation of TLR/ IL-1R and CD80 signaling pathways}

\section{Interaction with the MyD88 adap- tor complex}

IRAK-M can heterodimerize with IRAK-1 or 2 and bind both MyD88 and tumor necrosis factor receptor associated factor 6 (TRAF6). ${ }^{2}$ Upon TLR/IL-1R ligand engagement and formation of the MyD88 adaptor complex, IRAK-M is thought to bind MyD88/RAK-4 and inhibit IRAK-4 phosphorylation of IRAK-1. This prevents formation of TRAF6/IRAK-1 complexes, which initiate IKB kinase (IKK) and mitogen activated protein (MAP) kinase signaling pathways for activation of nuclear factor kappa $B$ (NF- $\mathrm{KB}$ ) and activator protein (AP-1) transcription factors. ${ }^{6,7}$ This role for IRAK-M in the negative regulation of TLR/IL-1R signaling was established in experiments demonstrating that transfection of MyD88 and IRAK-1 into 293T cells increases IRAK-1 phosphorylation. However, transfection of IRAK-M along with MyD88 and IRAK-1 increases the amount of unphosphorylated IRAK-1 relative to phosphorylated IRAK-1. Furthermore, overexpression of IRAK-M diminishes formation of IRAK1/TRAF6 complexes. ${ }^{7}$ The exact way in which IRAK-M inhibits NF- $\mathrm{B}$ and AP-1 activation likely depends on the particular stimulus.

\section{Inhibition of TLR-mediated NF-KB activation}

TLR signaling activates NF- $\mathrm{KB}$ via the classical or alternative pathway. In the classical pathway, the NF- $\kappa$ B complex (composed of the subunits $\mathrm{I} \kappa \mathrm{B} / \mathrm{p} 65 / \mathrm{p} 50$ ) is activated by IKK phosphorylation of the I $\mathrm{I}$ subunit. I $\mathrm{B}$ is ubiquitinated for proteosomal degradation, and the p65/p50 subunits translocate to the nucleus to bind pro-inflammatory gene pro-

\begin{tabular}{|c|c|c|}
\hline Inhibitor & Action & Source \\
\hline IRAK-M & $\begin{array}{l}\text { Inhibits IRAK-1 activation and formation } \\
\text { of IRAK-1/TRAF6 complexes }\end{array}$ & Kobayashi et al. ${ }^{7}$ \\
\hline IRAK-2c/-2d & IRAK-2 isoforms inhibit TLR-mediated NF- $\kappa B$ activation & Hardy et $a l^{4}$ \\
\hline Triad3A & Ubiquitinates TLRs for proteasomal degradation & Chuang et al. ${ }^{62}$ \\
\hline SOCS-1 & $\begin{array}{l}\text { Induces ubiquitination of MAL, which is necessary for } \\
\text { MyD88-dependent TLR-2 and -4 signaling }\end{array}$ & Mansell et al. ${ }^{63}$ \\
\hline PIN1 & $\begin{array}{l}\text { Inhibits activation of IRF3, a transcription factor } \\
\text { of TLR signaling }\end{array}$ & Saitoh et al..$^{64}$ \\
\hline A20 & Deubiquitinates and inhibits TRAF6 activity & Boone et al..$^{65}$ \\
\hline DUBA & Deubiquitinates and inhibits TRAF3 activity & Kayagaki et $a l^{66}$ \\
\hline CYLD & Deubiquitinates and inhibits TRAF6/7 activity & Yoshida et al. ${ }^{19}$ \\
\hline SARM & Competes with TRIF-signaling pathway & Carty et al. ${ }^{67}$ \\
\hline RP105 & Competes with LPS for interaction with TLR4 & Divanovic et al. ${ }^{68}$ \\
\hline MyD88s & Dominate negative inhibitor of TLR signaling & Janssens et al. ${ }^{99}$ \\
\hline TRAIL-R & 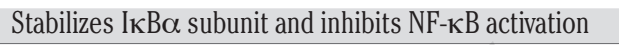 & Diehl et al. ${ }^{70}$ \\
\hline ATF3 & Inhibits NF-кB promoter interaction & Gilchrist et al. ${ }^{11}$ \\
\hline sTLR & $\begin{array}{l}\text { Soluble TLR competes with membrane bound TLRs } \\
\text { for ligand binding }\end{array}$ & Iwami et al. ${ }^{72}$ \\
\hline DC-SIGN & $\begin{array}{l}\text { Binds bacterial cell wall component Man-LAM, } \\
\text { inhibiting NF-אB and enhancing IL-10 production }\end{array}$ & Geijtenbeek et al. ${ }^{43}$ \\
\hline PI3K & $\begin{array}{l}\text { Activates AKT signaling pathway, which inhibits } \\
\text { TLR-mediated NF- } \kappa \text { B activation }\end{array}$ & 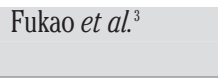 \\
\hline SHP-1 & Inhibits NF- $\kappa B$ and MAP kinase activation & An et $a l^{73}$ \\
\hline SHP-2 & Inhibits TRIF-dependent TLR-3 and -4 signaling & An et $a .^{74}$ \\
\hline
\end{tabular}

Table 2. IRAK-M expression pattern in cells and tissues.

\begin{tabular}{|c|c|c|}
\hline Cell or tissue expressing IRAK-M & Species & Source \\
\hline $\begin{array}{l}\text { Immune Cells } \\
\text { Monocytes/Macrophages } \\
\text { Neutrophils } \\
\text { Fibroblasts } \\
\text { B cells }\end{array}$ & $\begin{array}{l}\text { Human and Mouse } \\
\text { Mouse } \\
\text { Mouse } \\
\text { Mouse }\end{array}$ & $\begin{array}{l}\text { Wesche et al. }{ }^{2} \text { Rosati et } a l^{5}{ }^{5} \\
\text { Hubbard et al. } .^{11} \\
\text { Rosati et al. }{ }^{5} \\
\text { Meyer-Bahlburg et al. }{ }^{12}\end{array}$ \\
\hline $\begin{array}{l}\text { Epithelial Cells } \\
\text { Lung } \\
\text { Intestine } \\
\text { Intrahepatic bilary duct }\end{array}$ & $\begin{array}{l}\text { Human and Mouse } \\
\text { Mouse } \\
\text { Mouse }\end{array}$ & $\begin{array}{l}\text { Balaci et al. } .^{8} \text { Seki } \text { et } a l^{10} \\
\text { Oshima et al. }{ }^{14} \\
\text { Harada et al. }{ }^{13}\end{array}$ \\
\hline $\begin{array}{l}\text { Tissues } \\
\text { Thymus } \\
\text { Liver } \\
\text { Heart } \\
\text { Brain } \\
\text { Spleen } \\
\text { Kidney }\end{array}$ & Mouse & Rosati et al. ${ }^{5}$ \\
\hline
\end{tabular}

moter sequences. ${ }^{6}$ The alternative pathway involves activation of the NF- $\mathrm{B} 2$ complex (RelB/p100) by NFKB-inducing kinase (NIK). The p100 subunit is cleaved at the C-terminus generating p52, and RelB/p52 translocate to the nucleus to induce gene transcription. ${ }^{15,16}$ Kobayashi et al. demonstrated that IRAK-M can inhibit the classical pathway in a TLR-4 and -9 dependent manner.

However, endotoxin tolerance has been associated with decreased NF- $\kappa$ B2 levels, ${ }^{17}$ and there is evidence that IRAK-M can selectively inhibit the alternative NF- $\kappa$ B pathway in a TLR-2 dependent manner. ${ }^{18}$ Su et al. demonstrated that following TLR2 stimulation, the same level of RelA/p65 (classical pathway) nuclear translocation is observed in WT and IRAK-M -/- bone marrow-derived macrophages (BMDM); however, IRAK-M-/- BMDM display increased RelB (alternative pathway) nuclear translocation relative to WT BMDM. Furthermore, IRAK-M -/- BMDM display 
increased expression of NIK protein..$^{18}$ Overall, these data suggest that IRAK-M can negatively regulate either NF- $\mathrm{KB}$ pathway depending on the TLR stimulus.

\section{Inhibition of TLR-mediated AP-1 activation}

IRAK-M may also negatively regulate activation of the transcription factor AP-1 by inhibiting TLR-mediated MAP kinase activation. The synthetic TLR2 ligand, Pam3CSK4, is known to induce activation of the MAP kinases p38, extracellular signal-regulated kinase (ERK) $1 / 2$, and c-jun N-terminal kinase (JNK), which in turn can activate AP-1. ${ }^{19}$ However, Su et al. found that IRAK-M selectively attenuates p38 but not JNK or ERK1/2 following treatment with Pam3CSK4.$^{20}$ Furthermore, Kobayashi et al. demonstrated that IRAK-M inhibits CpG DNA- and LPS-induced p38 and ERK1/2 activation. CpG DNA activation of JNK is also inhibited by IRAK-M. ${ }^{7}$ These data suggest a possible mechanism for IRAK-M inhibition of AP-1 activation via inhibition of multiple MAP kinases.

\section{Regulation of CD80 signaling}

IRAK-M may bind and regulate activation of the costimulatory molecule CD80. In response to CD28 engagement, CD80 and CD86 expressed on APCs signal downstream activation of NF- $\mathrm{KB} / \mathrm{AP}-1 .^{21}$ In a mouse model of sepsis, CD80 appears to be the dominant receptor for regulating immune activation and lethality following early cecal ligation and puncture. ${ }^{22} \mathrm{As}$ determined by confocal microscopy, IRAK-M seems to interact with CD80 and disassociates in response to stimulation with CD28-containing neutrophil lipid rafts. This leads to decreased IRAK-M interataction with TRAF6 which may contribute to induction of lethal cytokine storm and pathological inflammation. ${ }^{22}$ These data suggest that IRAK-M may negatively regulate activation of NF- $\mathrm{KB} / \mathrm{AP}-1$ via CD80 signaling in addition to TLR/IL-1R signaling; however, further studies are needed to explore this possibility.

\section{Regulation of IRAK-M expression}

Expression of IRAK-M can be altered in response to a number of molecules. This includes cell surface and intracellular signaling molecules as well as endogenous and exogenous soluble factors. A summary of these molecules and their effect on IRAK-M expression are listed in Table 3 and are briefly summarized below.

\section{TLRs}

IRAK-M expression is induced in response to activation of a number of TLRs, as a means to generate tolerance to subsequent TLR stimulus. IRAK-M mRNA and protein are expressed in macrophages within 3 and $6 \mathrm{~h}$, respectively, following stimulation with LPS, an endotoxin component of bacterial cell walls that activates TLR4. ${ }^{7,23}$ TLR2 recognizes the bacterial cell wall components peptidoglycan (PGN) and lipoteichoic acid (LTA). IRAK-M expression is induced $4 \mathrm{~h}$ following PGN treatment. Furthermore, TNF- $\alpha$ production and activation of MAP kinases, I $\mathrm{B} \alpha$, and IRAK-1 are reduced following second PGN stimulation. Silencing IRAK-M restores TNF- $\alpha$ production in this setting. ${ }^{24}$ Treatment with LTA can also tolerize macrophages to LPS-induced activation of NF$\kappa \mathrm{B}$ as well as increase IRAK-M expression. ${ }^{25}$

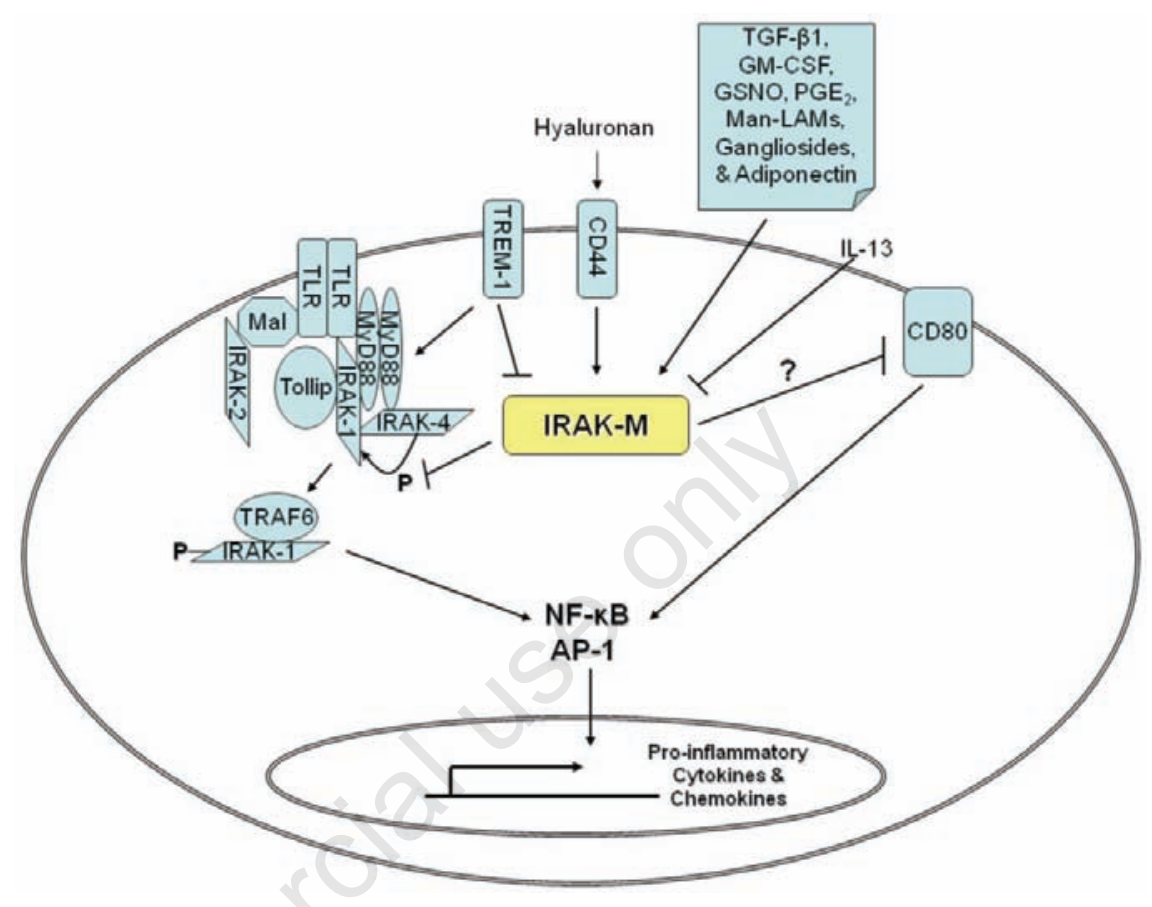

Figure 1. IRAK-M regulation and function in macrophage host defense.

Table 3. Regulators of IRAK-M expression.

Regulator IRAK-M expression Source

Soluble factors

Adiponectin

$\alpha$-melanocyte-stimulating hormone

$\mathrm{PGE}_{2}$

Gangliosides

Hyaluronan

GM-CSF

IL-13

TGF- $\beta 1$

Man-LAMs

GNSO

\begin{tabular}{|c|c|c|}
\hline \\
\hline $\begin{array}{l}\text { Intracellular signaling molecules } \\
\text { PI3K }\end{array}$ & Unclear & $\begin{array}{l}\text { Fukao et al. } \\
\text { Taylor } \mathrm{AW}^{33}\end{array}$ \\
\hline \multicolumn{3}{|l|}{ Cell surface receptors } \\
\hline TREM-1 & $\downarrow$ & Lagler et $a .^{9}$ \\
\hline TLR2 & $\uparrow$ & $\begin{array}{l}\text { Nakayama et al. } \\
\text { Kim et al. }{ }^{25}\end{array}$ \\
\hline TLR4 & $\uparrow$ & $\begin{array}{l}\text { Kobayashi et al. } \\
\text { Mages et al. }\end{array}$ \\
\hline TLR5 & $\uparrow$ & Oshima et al. ${ }^{14}$ \\
\hline TLR7 & $\uparrow$ & $\begin{array}{l}\text { Hassan et a }{ }^{26} \\
\text { Hayashi } \text { et al. }^{27}\end{array}$ \\
\hline TLR9 & $\uparrow$ & $\begin{array}{l}\text { Kim et al. } \\
\text { Tazi et al. }\end{array}$ \\
\hline
\end{tabular}

Zacharioudaki et al. ${ }^{33}$

Taylor AW ${ }^{31}$

Hubbard et al."

Shen et al..$^{34}$

Yatabe et al. ${ }^{35}$

Berclaz et al. ${ }^{38}$

Scotton et al. ${ }^{47}$

Pan et al. ${ }^{42}$

Pathak et al. ${ }^{46}$

Gonzalez-Leon et al..$^{39}$

del Fresno et al. ${ }^{40}$

\section{Fukao et al. ${ }^{3}$}

Lagler et al. ${ }^{9}$

Nakayama et al. ${ }^{24}$

Oshima et al.

Hassan et al ${ }^{22}$

Kim et al.
Tazi et al. ${ }^{29}$ 
A similar profile is observed in TLR7 tolerized cells, which display elevated IRAK-M expression, diminished production of TNF- $\alpha$, and reduced activation of $\mathrm{NF}-\kappa \mathrm{B}, \mathrm{p} 38$ and stress-activated protein kinase (SAPK) ${ }^{26}$ In a murine allergic encephalomyelitis model, a repeated low dose of intravenous TLR7 agonist reduces inflammation and induces tolerance to intranasally administered TLR-2, -7 , and -9 ligands. Induction of TLR7 tolerance involves increases in IRAK-M and SH2 domain containing protein-tyrosine phosphatase (SHP-1) expression. ${ }^{27}$ IRAK-M is also elevated in liver macrophages following low level CpG DNA (TLR9 ligand) treatment in vivo. ${ }^{28}$ In this model of liver injury, TNF- $\alpha$ secreted from activated macrophages in response to LPS and CpG DNA treatment induces hepatocyte apoptosis. However, macrophages become hyporesponsive to CpG DNA-induced injury following pretreatment with CpG DNA. This not only requires elevation of IRAK-M but decreases in IRAK-1 expression. ${ }^{28}$ Thus, tolerization associated with elevated IRAK-M likely serves to prevent liver cell death. In cirrhotic monocytes, LPS-induced TNF- $\alpha$ production is associated with a lack of IRAK-M induction. This lack of IRAK-M induction may promote ongoing liver injury but may also serve as a means to sensitize cirrhotic monocytes to pathogenic stimuli. ${ }^{29}$ Lastly, the TLR5 ligand, flagellin, induces IRAK-M expression in macrophages $8 \mathrm{~h}$ posttreatment, and this induction of IRAK-M is also associated with tolerance..$^{14}$ Overall, induction of IRAK-M is necessary to limit pathologic inflammation and cytokine secretion.

\section{Intracellular signaling and cell surface molecules}

Expression of IRAK-M is preceded by induction of other negative regulators of TLR/L-1R signaling, such as PI3K, ${ }^{3,14}$ but it is not clear how exactly PI3K may influence IRAK-M expression. Escoll et al. demonstrated that IRAK-M mRNA in septic monocytes is more rapidly induced in the presence of a PI3K inhibitor. ${ }^{30}$ Conversely, induction of IRAK-M expression by adiponectin requires activation of PI3K. ${ }^{31}$

IRAK-M expression can also be regulated by activation of cell surface molecules, such as triggering receptor expressed on myeloid cells 1 (TREM-1). Lagler et al. demonstrated that TREM-1 activation during early Streptococcus pneumoniae infection results in decreased lung IRAK-M expression and elevated pro-inflammatory cytokines. ${ }^{9}$ Their data suggests that this decrease in IRAK-M expression mediated by TREM-1 promotes efficient early bacterial clearance. It is interesting that TREM-1 activation may be able to modulate the early IRAK-M activation signals that are normally triggered by TLRs in response to bacterial infection.

\begin{tabular}{|c|c|c|}
\hline Disease & $\begin{array}{l}\text { Phenotype associated with } \\
\text { increased IRAK-M expression }\end{array}$ & $\begin{array}{l}\text { Phenotype associated with } \\
\text { decreased IRAK-M expression }\end{array}$ \\
\hline Sepsis & $\begin{array}{l}\text { Induction of macrophage } \\
\text { endotoxin tolerance and reduced } \\
\text { proinflammatory cytokine } \\
\text { production (toxic shock). } 4 ., 54,55\end{array}$ & $\begin{array}{l}\text { Increased cytokine production, } \\
\text { increased neutrophil recruitment } \\
\text { to site of infection, and reduced } \\
\text { susceptibility to secondary infection. }{ }^{55}\end{array}$ \\
\hline $\begin{array}{l}\text { Inflammatory } \\
\text { bowel disease }\end{array}$ & $?$ & $\begin{array}{l}\text { Lack of tolerance to commensal } \\
\text { gastrointestinal bacteria. }{ }^{49,50}\end{array}$ \\
\hline Allograft tolerance & $?$ & $\begin{array}{l}\text { Reduced tolerance to skin allografts } \\
\text { and reduced effectiveness } \\
\text { of exogenous costimulatory } \\
\text { molecule inhibition. }^{51}\end{array}$ \\
\hline Influenza infection & $?$ & $\begin{array}{l}\text { Increased neutrophil recruitment to } \\
\text { the lung and no effect on viral } \\
\text { clearance. }^{10}\end{array}$ \\
\hline $\begin{array}{l}\text { P. gingivalis peridontal } \\
\text { infection }\end{array}$ & $\begin{array}{l}\text { Induction of macrophage endotoxin } \\
\text { tolerance and reduced immune } \\
\text { responsiveness. }\end{array}$ & $?$ \\
\hline Tumor development & $\begin{array}{l}\text { Monocyte deactivation and reduced } \\
\text { immune responsiveness. }{ }^{6}\end{array}$ & $\begin{array}{l}\text { Reduced tumor growth and enhanced } \\
\text { anti-tumor immunity. }\end{array}$ \\
\hline $\begin{array}{l}\text { Hematopoietic stem } \\
\text { cell transplant related } \\
\text { infections }\end{array}$ & $\begin{array}{l}\text { Reduced bacterial clearance following } \\
\text { acute lung infection and reduced } \\
\text { macrophage } \\
\text { host defense." }\end{array}$ & $\begin{array}{l}\text { Restored bacterial } \\
\text { clearance following acute lung } \\
\text { infection and enhanced macrophage } \\
\text { host defense." }\end{array}$ \\
\hline
\end{tabular}

\section{Soluble factors}

The protein hormone, adiponectin, is secreted by adipocytes and has anti-inflammatory properties. ${ }^{32}$ Induction of IRAK-M expression by adiponectin requires activation of phosphatidyl inositol 3-kinase (PI3K), protein kinase B (AKT/PKB), and ERK and induces macrophage endotoxin tolerance. ${ }^{31}$ However, it is not known what transcription factors are activated in response to adiponectin treatment that induce expression of IRAK-M. IRAK-M may also play an inhibitory role in macrophages treated with $\alpha$-melanocyte-stimulating horomone (MSH). ${ }^{33}$ LPS-stimulated macrophages treated with $\alpha$-MSH are suppressed in both nitric oxide and IL-12p70 production and cannot promote Th1 cell interferon (IFN)- $\gamma$ production. In these tolerant cells, IRAK-M is bound to IRAK-1, suggesting a role for IRAK-M in $\alpha$-MSH induced macrophage tolerance. $^{33}$

Factors produced by certain tumors can induce immunosuppression in the tumor environment by upregulating macrophage expression of IRAK-M. For example, gangliosides are sialic acid-containing glycosphingolipids in the outer plasma membrane and are produced in high amount by tumors. These molecules can increase IRAK-M expression and inhibit TLR signaling in monocytes. ${ }^{34}$ Hyaluronan, a glycosaminoglycan in the extracellular matrix, may also have a similar effect, as HA2700 was shown to upregulate IRAK-M expression in chondrocytes. ${ }^{35}$ Evidence suggests that this may be via CD44 and/or TLR4 intermediates. ${ }^{36}$
Our own work suggests that IRAK-M can also be upregulated by prostaglandin E2 (PGE2), a soluble lipid mediator known to be overproduced in many cancers and immunodeficient states. ${ }^{11,37}$

Berclaz et al. found that IRAK-M expression is dependent upon granulocyte-macrophage colony stimulating factor (GM-CSF) and expression of the transcription factor, PU.1. ${ }^{38}$ These data are the first to identify a transcription factor regulating the expression of IRAKM. In addition to GM-CSF, IRAK-M expression may also require TNF- $\alpha$. The nitric oxide donor molecule, S-Nitrosoglutathione (GSNO), is known to induce IRAK-M expression in human monocytes and this requires TNF- $\alpha{ }^{39,40}$ Furthermore, LPS fails to induce IRAK-M expression, following in vitro antibody depletion of whole blood leukocyte TNF- $\alpha{ }^{41}$

Pan et al. showed that LPS-induced IRAK-M upregulation may also require activation of the transforming growth factor (TGF)- $\beta 1$ signaling pathway. ${ }^{42}$ TGF- $\beta 1$ increases following LPS treatment and can upregulate IRAK-M and Src homology 2 domain-containing inositol 5phosphatase-1 (SHIP1) expression in a SMAD4 dependent manner. Furthermore, silencing SMAD4 or SHIP1 expression inhibits TGF- $\beta$-mediated IRAK-M elevation. ${ }^{42}$

Mannose receptors and the $\mathrm{C}$-type lectin dendritic cell-specific intercellular adhesion molecule-3-grabbing non-integrin (DC-SIGN) are known to bind Mycobacterium tuberculosis cell wall components called mannose-capped lipoarabinomannans (Man-LAMs). ${ }^{43-45}$ Pathak et 
al. demonstrated that Man-LAMs inhibit LPSinduced IL-12 $\mathrm{p} 40$ production, NF- $\mathrm{KB}$ activation, and IRAK-1/TRAF6 interaction. ${ }^{46}$ This is accompanied by increased IRAK-M expression. IRAKM may mediate Man-LAM induced macrophage deactivation, as silencing of IRAK-M restores LPS-induced IL-12 production in Man-LAM treated cells. It is assumed that the mannooligosaccharide caps of LAM are the main factors responsible for induction of IRAK-M. ${ }^{46}$

Lastly, the alternatively activated macrophage phenotype may be associated with the downregulation of IRAK-M expression. IL-13 and IL- 4 are Th2 cytokines known to induce this phenotype in macrophages, and it was determined that expression of IRAK-M was decreased in response to IL-13 treatment. ${ }^{47}$

\section{Clinical scenarios in which IRAK-M expression is beneficial}

IRAK-M has varying roles in immunopathology depending on the disease context (Table 4). For example, in the setting of chronic inflammatory diseases, IRAK-M expression is desirable because it can limit excessive immune responses. In contrast, IRAK-M expression may prevent proper innate immune clearance of pathogens in the setting of immunodeficiency. Thus, from a clinical perspective, it is useful to understand situations in which modulation of IRAK-M expression may offer therapeutic benefit.

\section{Sepsis and endotoxin tolerance in macrophages}

In response to overwhelming infection or gut damage, endotoxins, such as LPS, are released from bacteria. Prolonged secretion of these mediators can result in septic shock and multi-organ failure. However, monocytic cells develop tolerance to constant exposure to or re-challenge with LPS to limit toxic shock or excessive systemic inflammation. Endotoxin tolerance is associated with reduced IRAK-1 and NF- $\kappa \mathrm{B}$ activation, downregulation of proinflammatory cytokine gene transcription, and upregulation of anti-inflammatory mediators, such as IL-10 and TGF- $\beta{ }^{48}$ Antigen presentation is reduced as a result of decreased expression of HLA-DR and phagocytosis is enhanced as scavenger receptors such as MARCO increase. ${ }^{17}$ A number of regulatory mediators are induced during this response; however, rapid elevation in IRAK-M expression has become a sentinel feature of the induction of endotoxin tolerance. Following induction of IRAK-M expression, IRAK-M mediates endotoxin tolerance in monocytic cells by inhibiting further TLR-mediated NF- $\mathrm{BB}$ and AP-1 activa- tion. ${ }^{6,7}$ Accordingly, macrophages deficient in IRAK-M expression have elevated pro-inflammatory cytokine production in response to TLR stimulus. ${ }^{7}$ Therefore, induction of IRAK-M in early sepsis may be beneficial to the host by limiting septic shock and subsequent organ failure. As detailed in a subsequent section, however, the benefit on patient outcome is largely determined by the degree of IRAK-M induction as well as the ability of monocytic cells to respond to any secondary infection that may occur during the refractory or immunosuppressive stage of sepsis.

\section{Inflammatory bowel disease}

Commensal colonization of the gastrointestinal (GI) tract is generally not associated with immune activation. This likely reflects the fact that the host establishes tolerance against normal GI flora. Takebayashi et al. demonstrated that IRAK-M expression may regulate this process. ${ }^{49}$ Intraluminal injection of LPS enhances adherence of T cells to the intestinal epithelium of germ-free mice but not specific pathogen free (SPF) mice. SPF mice show increased IRAK-M and TGF- $\beta$ mRNA expression following LPS suggesting that tolerance induction is mediated by both of these molecules. ${ }^{49}$ Moreover, deficiencies in IRAK-M may predispose individuals to inflammatory bowel disease (IBD), as IRAK-M is on the genetic susceptibility locus for IBD ${ }^{50}$ In ulcerative colitis patients, an association between caspase recruitment domain (CARD15) mutant patients and IRAK-M was found, suggesting a possible impairment in the negative regulation of TLR-signaling causing IBD. ${ }^{50}$

\section{Allograft acceptance}

The role of IRAK-M in immune tolerance may also extend to allograft acceptance in the transplanted host. In a skin allograft model, IRAK-M -/- mice have elevated IL-6 and TNF- $\alpha$, which inhibits allograft acceptance. Furthermore, the costimulatory blockade regimen used to prolong graft survival is impaired in the absence of IRAK-M. ${ }^{51}$ This suggests that factors mediating immune tolerance to the allograft may induce expression of IRAK-M in certain cell types; however, it was not determined which cells expressing IRAK-M may mediate this acceptance. Determining whether the effect is mediated by leukocytes or resident structural cells may inform efforts to enhance IRAK-M expression in a therapeutic fashion and promote allograft acceptance.

\section{Influenza infection and asthma}

Influenza infection causes host damage not only through lytic destruction of lung epithelial cells but also via inappropriate immune cell activation post-infection. ${ }^{52}$ A murine model of influenza infection suggests that IRAK-M expression may limit immunopathology without decreasing viral clearance. ${ }^{10}$ Following influenza infection, IRAK-M increases in the lung 2 days post-infection and diminishes around day 7 . Interestingly, IRAK-M deficiency does not improve viral clearance, and neutrophil recruitment to the lung is increased in IRAK-M-/- mice, causing tissue damage. ${ }^{10}$ It is also possible that IRAK-M protects from complications of asthma. IRAK-M is highly expressed in lung epithelial cells and inactivation mutations were found in the IRAK-M coding region, which is located in the asthma susceptibility region $12 q 13-14 .^{8}$ Potentially, expression of IRAK-M may limit neutrophilinduced airway damage and remodeling.

\section{Clinical scenarios in which IRAK-M expression is detrimental}

\section{Peridontal infection}

While IRAK-M may serve an important role in limiting inappropriate immune activation, there are scenarios in which elevations in IRAK-M prevent appropriate host defense (Table 4). For example, Porphyromonas gingivalis, an infectious agent in peridontal tissue infection, induces a dampened immune response relative to $E$. coli and may employ IRAK-M induction as a means of escape from immune surveillance. ${ }^{53} P$. gingivalis LPS treated macrophages have increased induction of IRAK-M and degradation of IRAK-1 compared to $E$. coli LPS treated cells. Furthermore, silencing IRAK-M expression restores proinflammatory cytokine production in $P$. gingivalis LPS treated cells. Thus, clearance of this particular pathogen may be improved if IRAK$\mathrm{M}$ expression were therapeutically reduced.

\section{Secondary infection following sepsis}

Induction of IRAK-M expression may serve to limit septic shock and facilitate macrophage deactivation in early sepsis. However, higher levels of IRAK-M mRNA expression in admitted patient whole blood correlate with increased mortality. ${ }^{54}$ Furthermore, macrophage deactivation renders patients more susceptible to secondary infection. In a mouse model of sepsis, Deng et al. demonstrated that following secondary challenge with Pseudomonas aeruginosa lung infection, septic IRAK-M -/- mice have decreased mortality, improved bacterial clearance, elevated production of pro-inflammatory cytokines and chemokines, and increased neutrophil recruitment to the lung. ${ }^{55}$ Thus, deficiency in IRAK-M can improve the ability of septic mice to resolve secondary 
infection. Taken together, these data suggest that sepsis is a disease where patient survival is greatly affected by the degree of IRAK-M induction and the duration of macrophage deactivation following infection.

\section{Tumor-induced immunosuppression}

In many cancers, the tumor microenvironment is immunosuppressive, and this may involve induction of IRAK-M in tumor-associated macrophages. Blood monocytes from patients with chronic myeloid leukemia and patients with metastasis have elevated IRAKM. Moreover, monocytes co-cultured with tumor cells or tumor cell supernatant show significantly decreased expression levels of TNF$\alpha$, IL-12, and IRAK-1 and increased expression of IRAK-M. ${ }^{56}$ In addition, Xie et al. showed that IRAK-M -/- mice are resistant to melonoma and fibrosarcoma tumor growth following tumor inoculation. This is likely due to the enhanced anti-tumor effector function of lymphocytes in the absence of IRAK-M, given that T cells and B cells display elevated proliferation and activation in IRAK-M -/- mice as well as enhanced NF- $\kappa$ B activation..$^{57}$ Thus, targeted depletion of IRAK-M serves to increase anti-tumor immune responses. Overall, these data provide further evidence that factors produced in the tumor microenvironment may require IRAK-M to induce immunosuppression.

\section{Infectious complications of hematopoietic stem cell transplant}

Following immune reconstitution, HSCT patients display impaired pulmonary host defense, rendering them more susceptible to bacterial, fungal and viral infections. This can occur both in the setting of autologous or allogeneic transplant. ${ }^{58,59}$ Our laboratory has previously demonstrated that mice undergoing syngeneic bone marrow transplant (BMT) have elevated levels of prostaglandin $\mathrm{E}_{2}\left(\mathrm{PGE}_{2}\right)$ in the lung, resulting in increased susceptibility to pulmonary $P$. aeruginosa infection and reduced alveolar macrophage function. ${ }^{60,61} \mathrm{We}$ have shown that $\mathrm{PGE}_{2}$ can induce IRAK-M expression in alveolar macrophages, and that inhibition of endogenous $\mathrm{PGE}_{2}$ production reduces IRAK-M elevation and improves bacterial phagocytosis and killing in alveolar macrophages. ${ }^{11,60}$ Additionally, transplanting IRAK-M -/- bone marrow into WT mice enhances alveolar macrophage host defense function and improves bacterial clearance following $P$. aeruginosa infection, despite $\mathrm{PGE}_{2}$ elevation. ${ }^{11}$ These data strongly suggest that the wellknown inhibitory actions of $\mathrm{PGE}_{2}$ on alveolar macrophage function may require IRAK-M induction. Furthermore, these data suggest that therapeutic targeting of IRAK-M in the setting of bacterial infection post-HSCT may improve the ability of the host to phagocytose and kill bacterial pathogens.

\section{Conclusions}

IRAK-M has a well-established role in reducing immune responsiveness to continuous pathogen exposure. By negatively regulating TLR signaling, IRAK-M inhibits production of pro-inflammatory mediators and contributes to the induction of endotoxin tolerance. Furthermore, it is now appreciated that IRAK$M$ is expressed in multiple immune and epithelial cells types, and IRAK-M can be induced by a variety of stimuli. This suggests that IRAK-M may play a role in regulating a wider range of inflammatory conditions than previously thought. Despite these recent studies regarding IRAK-M expression and function, little is known about its transcriptional regulation. Future studies are needed to identify transcriptional regulators which induce or inhibit IRAK-M transcription.

IRAK-M remains an interesting target to consider for therapeutic manipulation. Induction of IRAK-M expression in certain settings may serve to limit pathologies associated with excessive cytokine production and innate immune activation. However, specific targeting and limited duration of IRAK-M induction must be employed to minimize host susceptibility to infection. In addition, clinical scenarios associated with increased susceptibility to infection may be improved by inhibiting IRAK$\mathrm{M}$ expression. However, it is not known what degree of suppression of IRAK-M may provide therapeutic benefit. Future studies employing RNA silencing technologies are needed to determine what degree of IRAK-M silencing would restore appropriate immune responsiveness. Moreover, IRAK-M is expressed in multiple cell types and induction or silencing of IRAK-M may need to be cell specific. Overall, the pleiotropic effects of IRAK-M make it an attractive target for therapeutic manipulation to improve the clinical management of patients with a variety of immune-related disorders.

\section{References}

1. Wang J, Hu Y, Deng WW, Sun B. Negative regulation of Toll-like receptor signaling pathway. Microbes Infect 2009;11:321-7.

2. Fukao T, Koyasu S. PI3K and negative regulation of TLR signaling. Trends Immunol 2003;24:358-63.

3. Wesche H, Gao X, Li X et al. IRAK-M is a novel member of the Pelle/interleukin-1 receptor-associated kinase (IRAK) family. J Biol Chem 1999;274:19403-10.

4. Hardy MP, O'Neill LA. The murine IRAK2 gene encodes four alternatively spliced isoforms, two of which are inhibitory. J
Biol Chem 2004;279:27699-708.

5. Rosati 0, Martin MU. Identification and characterization of murine IRAK-M. Biochem Biophys Res Commun 2002; 293:1472-7.

6. Janssens S, Beyaert R. Functional diversity and regulation of different interleukin-1 receptor-associated kinase (IRAK) family members. Mol Cell 2003;11:293-302.

7. Kobayashi K, Hernandez LD, Galan JE et al. IRAK-M is a negative regulator of Tolllike receptor signaling. Cell 2002;110:191202.

8. Balaci L, Spada MC, Olla N et al. IRAK-M is involved in the pathogenesis of early-onset persistent asthma. Am J Hum Genet 2007; 80:1103-14.

9. Lagler H, Sharif 0, Haslinger I et al. TREM1 activation alters the dynamics of pulmonary IRAK-M expression in vivo and improves host defense during pneumococcal pneumonia. J Immunol 2009;183:202736.

10. Seki M, Kohno S, Newstead MW et al. Critical role of IL-1 receptor-associated kinase-M in regulating chemokinedependent deleterious inflammation in murine influenza pneumonia. J Immunol 2010;184:1410-8.

11. Hubbard LL, Ballinger MN, Thomas PE et al. A role for IL-1 Receptor-associated kinase-M in Prostaglandin E(2)-induced immunosuppression post-bone marrow transplantation. J Immunol 2010;184:6299308.

12. Meyer-Bahlburg A, Khim S, Rawlings DJ. B cell intrinsic TLR signals amplify but are not required for humoral immunity. J Exp Med 2007;204:3095-101.

13. Harada K, Nakanuma Y. Biliary innate immunity and cholangiopathy. Hepatol Res 2007;37:S430-7.

14. Oshima N, Ishihara S, Rumi MA et al. A20 is an early responding negative regulator of Toll-like receptor 5 signalling in intestinal epithelial cells during inflammation. Clin Exp Immunol 2010;159:185-98.

15. Neumann M, Naumann M. Beyond IkappaBs: alternative regulation of NFkappaB activity. FASEB J 2007;21:2642-54.

16. Xiao G, Rabson AB, Young $W$ et al. Alternative pathways of NF-kappaB activation: a double-edged sword in health and disease. Cytokine Growth Factor Rev 2006; 17:281-93.

17. del Fresno C, Garcia-Rio F, Gomez-Pina V et al. Potent phagocytic activity with impaired antigen presentation identifying lipopolysaccharide-tolerant human monocytes: demonstration in isolated monocytes from cystic fibrosis patients. J Immunol 2009;182:6494-507.

18. Su J, Zhang T, Tyson J, Li L. The Interleukin-1 Receptor-Associated Kinase 
M Selectively Inhibits the Alternative, Instead of the Classical NFkappaB Pathway. J Innate Immun 2009;1:164-74.

19. Yoshida H, Jono H, Kai H, Li JD. The tumor suppressor cylindromatosis (CYLD) acts as a negative regulator for toll-like receptor 2 signaling via negative cross-talk with TRAF6 AND TRAF7. J Biol Chem 2005;280: 41111-21.

20. Su J, Xie Q, Wilson I, Li L. Differential regulation and role of interleukin-1 receptor associated kinase-M in innate immunity signaling. Cell Signal 2007;19:1596-601.

21. Orabona C, Grohmann U, Belladonna ML et al. CD28 induces immunostimulatory signals in dendritic cells via CD80 and CD86. Nat Immunol 2004;5:1134-42.

22. Nolan A, Kobayashi H, Naveed B et al. Differential role for CD80 and CD86 in the regulation of the innate immune response in murine polymicrobial sepsis. PLoS One 2009;4:e6600.

23. Mages J, Dietrich H, Lang R. A genomewide analysis of LPS tolerance in macrophages. Immunobiology 2007;212: 723-37.

24. Nakayama K, Okugawa S, Yanagimoto S et al. Involvement of IRAK-M in peptidoglycan-induced tolerance in macrophages. $\mathrm{J}$ Biol Chem 2004;279:6629-34.

25. Kim HG, Kim NR, Gim MG et al. Lipoteichoic acid isolated from Lactobacillus plantarum inhibits lipopolysaccharide-induced TNF-alpha production in THP-1 cells and endotoxin shock in mice. J Immunol 2008;180:2553-61.

26. Hassan F, Islam S, Tumurkhuu $\mathrm{G}$ et al. Involvement of interleukin-1 receptorassociated kinase (IRAK)-M in toll-like receptor (TLR) 7-mediated tolerance in RAW 264.7 macrophage-like cells. Cell Immunol 2009;256:99-103.

27. Hayashi T, Gray CS, Chan M et al. Prevention of autoimmune disease by induction of tolerance to Toll-like receptor 7. Proc Natl Acad Sci USA 2009;106:2764-9.

28. Kim YI, Park JE, Martinez-Hernandez A, Yi AK. CpG DNA prevents liver injury and shock-mediated death by modulating expression of interleukin-1 receptor-associated kinases. J Biol Chem 2008;283: 15258-70.

29. Tazi KA, Quioc JJ, Saada V et al. Upregulation of TNF-alpha production signaling pathways in monocytes from patients with advanced cirrhosis: possible role of Akt and IRAK-M. J Hepatol 2006; 45:280-9.

30. Escoll P, del Fresno C, Garcia L et al. Rapid up-regulation of IRAK-M expression following a second endotoxin challenge in human monocytes and in monocytes isolated from septic patients. Biochem Biophys Res Commun 2003;311:465-72.
31. Zacharioudaki V, Androulidaki A, Arranz A et al. Adiponectin promotes endotoxin tolerance in macrophages by inducing IRAKM expression. J Immunol 2009;182:644451.

32. Stofkova A. Leptin and adiponectin: from energy and metabolic dysbalance to inflammation and autoimmunity. Endocr Regul 2009;43:157-68.

33. Taylor AW. The immunomodulating neuropeptide alpha-melanocyte-stimulating hormone (alpha-MSH) suppresses LPSstimulated TLR4 with IRAK-M in macrophages. J Neuroimmunol 2005;162: 43-50.

34. Shen W, Stone K, Jales A et al. Inhibition of TLR activation and up-regulation of IL-1Rassociated kinase-M expression by exogenous gangliosides. J Immunol 2008;180: 4425-32.

35. Yatabe T, Mochizuki S, Takizawa $\mathrm{M}$ et al. Hyaluronan inhibits expression of ADAMTS4 (aggrecanase-1) in human osteoarthritic chondrocytes. Ann Rheum Dis 2009;68:1051-8.

36. del Fresno C, Otero K, Gomez-Garcia L et al. Tumor cells deactivate human monocytes by up-regulating IL-1 receptor associated kinase-M expression via CD44 and TLR4. J Immunol 2005;174:3032-40.

37. Greenhough A, Smartt HJ, Moore AE et al. The COX-2/PGE2 pathway: key roles in the hallmarks of cancer and adaptation to the tumour microenvironment. Carcinogenesis 2009;30:377-86.

38. Berclaz PY, Carey B, Fillipi MD et al. GMCSF Regulates a PU.1-Dependent Transcriptional Program Determining the Pulmonary Response to LPS. Am J Respir Cell Mol Biol 2007;36:114-21.

39. Gonzalez-Leon MC, Soares-Schanoski A, del Fresno $\mathrm{C}$ et al. Nitric oxide induces S0CS-1 expression in human monocytes in a TNF-alpha-dependent manner. $\mathrm{J}$ Endotoxin Res 2006;12:296-306.

40. del Fresno C, Gomez-Garcia L, Caveda L et al. Nitric oxide activates the expression of IRAK-M via the release of TNF-alpha in human monocytes. Nitric Oxide 2004; 10:213-20.

41. van 't Veer $\mathrm{C}$, van den Pangaart PS, van Zoelen MA et al. Induction of IRAK-M is associated with lipopolysaccharide tolerance in a human endotoxemia model. $\mathrm{J}$ Immunol 2007;179:7110-20.

42. Pan H, Ding E, Hu M. SMAD4 is required for development of maximal endotoxin tolerance. J Immunol 2010;184:5502-9.

43. Geijtenbeek TB, Van Vliet SJ, Koppel EA et al. Mycobacteria target DC-SIGN to suppress dendritic cell function. J Exp Med 2003;197:7-17.

44. Maeda N, Nigou J, Herrmann JL et al. The cell surface receptor DC-SIGN discrimi- nates between Mycobacterium species through selective recognition of the mannose caps on lipoarabinomannan. J Biol Chem 2003;278:5513-6.

45. Nigou J, Zelle-Rieser C, Gilleron M et al. Mannosylated lipoarabinomannans inhibit IL-12 production by human dendritic cells: evidence for a negative signal delivered through the mannose receptor. J Immunol 2001;166:7477-85.

46. Pathak SK, Basu S, Bhattacharyya A et al. Mycobacterium tuberculosis lipoarabinomannan-mediated IRAK-M induction negatively regulates Toll-like receptor-dependent interleukin-12 p40 production in macrophages. J Biol Chem 2005;280: 42794-800.

47. Scotton CJ, Martinez FO, Smelt MJ et al. Transcriptional profiling reveals complex regulation of the monocyte IL-1 beta system by IL-13. J Immunol 2005;174:834-45.

48. Biswas SK, Lopez-Collazo E. Endotoxin tolerance: new mechanisms, molecules and clinical significance. Trends Immunol 2009;30:475-87.

49. Takebayashi K, Hokari R, Kurihara $\mathrm{C}$ et al. Oral tolerance induced by enterobacteria altered the process of lymphocyte recruitment to intestinal microvessels: roles of endothelial cell adhesion molecules, TGFbeta and negative regulators of TLR signaling. Microcirculation 2009;16:251-64.

50. Weersma RK, Oostenbrug LE, Nolte IM et al. Association of interleukin-1 receptorassociated kinase M (IRAK-M) and inflammatory bowel diseases. Scand J Gastroenterol 2007;42:827-33.

51. Shen H, Goldstein DR. IL-6 and TNF-alpha synergistically inhibit allograft acceptance. J Am Soc Nephrol 2009;20:1032-40.

52. Sun K, Metzger DW. Inhibition of pulmonary antibacterial defense by interferon-gamma during recovery from influenza infection. Nat Med 2008;14:558-64.

53. Domon H, Honda T, Oda T, et al. Early and preferential induction of IL-1 receptorassociated kinase-M in THP-1 cells by LPS derived from Porphyromonas gingivalis. J Leukoc Biol 2008;83:672-9.

54. Wiersinga WJ, van't Veer $\mathrm{C}$, van den Pangaart PS et al. Immunosuppression associated with interleukin-1R-associated-kinase-M upregulation predicts mortality in Gram-negative sepsis (melioidosis). Crit Care Med 2009;37:569-76.

55. Deng JC, Cheng G, Newstead MW et al. Sepsis-induced suppression of lung innate immunity is mediated by IRAK-M. J Clin Invest 2006;116:2532-42.

56. del Fresno C, Otero K, Gomez-Garcia L et al. Tumor cells deactivate human monocytes by up-regulating IL-1 receptor associated kinase-M expression via CD44 and TLR4. J Immunol 2005;174:3032-40. 
57. Xie Q, Gan L, Wang $\mathrm{J}$ et al. Loss of the innate immunity negative regulator IRAK$M$ leads to enhanced host immune defense against tumor growth. Mol Immunol 2007;44:3453-61.

58. Auletta JJ, Lazarus HM. Immune restoration following hematopoietic stem cell transplantation: an evolving target. Bone Marrow Transplant 2005;35:835-57.

59. Kotloff R, Ahya V, Crawford SW. Pulmonary complications of solid organ and hematopoietic stem cell transplantation. Am J Respir Crit Care Med 2004;170:22-48.

60. Ballinger MN, Aronoff DM, McMillan TR, et al. Critical Role of Prostaglandin E2 Overproduction in Impaired Pulmonary Host Response Following Bone Marrow Transplantation. J Immunol 2006;177: 5499-508.

61. Ojielo C, Cooke KR, Mancuso P et al. Defective phagocytosis and clearance of Pseudomonas aeruginosa in the Lung Following Bone Marrow Transplantation. J Immunol 2003;171:4416-24.

62. Chuang TH, Ulevitch RJ. Triad3A, an E3 ubiquitin-protein ligase regulating Tolllike receptors. Nat Immunol 2004;5:495502.
63. Mansell A, Smith R, Doyle SL et al. Suppressor of cytokine signaling 1 negatively regulates Toll-like receptor signaling by mediating Mal degradation. Nat Immunol 2006;7:148-55.

64. Saitoh T, Tun-Kyi A, Ryo A et al. Negative regulation of interferon-regulatory factor 3-dependent innate antiviral response by the prolyl isomerase Pin1. Nat Immunol 2006;7:598-605.

65. Boone DL, Turer EE, Lee EG et al. The ubiquitin-modifying enzyme A20 is required for termination of Toll-like receptor responses. Nat Immunol 2004;5:105260.

66. Kayagaki N, Phung Q, Chan S et al. DUBA: a deubiquitinase that regulates type I interferon production. Science 2007;318: 1628-32.

67. Carty M, Goodbody R, Schroder M et al. The human adaptor SARM negatively regulates adaptor protein TRIF-dependent Toll-like receptor signaling. Nat Immunol 2006;7:1074-81.

68. Divanovic S, Trompette A, Atabani SF et al. Negative regulation of Toll-like receptor 4 signaling by the Toll-like receptor homolog RP105. Nat Immunol 2005;6:571-8.
69. Janssens S, Burns K, Tschopp J, Beyaert R. Regulation of interleukin-1- and lipopolysaccharide-induced NF-kappaB activation by alternative splicing of MyD88. Curr Biol 2002;12:467-71.

70. Diehl GE, Yue HH, Hsieh K et al. TRAIL-R as a negative regulator of innate immune cell responses. Immunity 2004;21:877-89.

71. Gilchrist M, Thorsson V, Li B et al. Systems biology approaches identify ATF3 as a negative regulator of Toll-like receptor 4 . Nature 2006;441:173-8.

72. Iwami KI, Matsuguchi T, Masuda A et al. Cutting edge: naturally occurring soluble form of mouse Toll-like receptor 4 inhibits lipopolysaccharide signaling. J Immunol 2000;165:6682-6.

73. An H, Hou J, Zhou J et al. Phosphatase SHP-1 promotes TLR- and RIG-I-activated production of type I interferon by inhibiting the kinase IRAK1. Nat Immunol 2008; 9:542-50.

74. An H, Zhao W, Hou J et al. SHP-2 phosphatase negatively regulates the TRIF adaptor protein-dependent type I interferon and proinflammatory cytokine production. Immunity 2006;25:919-28. 